


\section{Geschichte der Sammlung}

Beinahe hätte die Münzsammlung der UB Leipzig schon im Frühjahr 2017 ihr 300-jähriges Jubiläum feiern können, denn am 12. Mai 1717 feierte August der Starke seinen 48. Geburtstag in Leipzig, wobei Vertreter der Universität ein Lobgedicht übergaben. Als Dank überreichte August eine Gold- und eine Silbermedaille, die goldene allein zu 50 Dukaten. Das kostbare Geschenk wurde allerdings nicht zum Ausgangspunkt einer numismatischen Sammlung: Schon 1718 veräußerte man die Medaillen wieder, der stattliche Erlös von 133 Talern und 8 Groschen finanzierte mit seinem Zinsertrag in den Folgejahren Erwerbungen der Universitätsbibliothek.

Im selben Jahr erhielt die Universität aber erneut ein Geschenk, das aus heutiger Rückschau den Grundstein für eine eigenständige Münzsammlung legte. Es umfasste einen Schatzfund von mitteldeutschen Brakteaten aus dem späteren 13. Jahrhundert und verdankte sich dem niederlausitzischen Studenten Christian Bernds. Bernds hatte sich erst im Wintersemester 1717 in Leipzig immatrikuliert. Was den Studenten in seinem zweiten Semester dazu gebracht hat, einen offenbar umfangreichen Silbermünzenfund seiner Alma Mater zu schenken, ist heute nicht mehr nachvollziehbar, da keine zeitgenössischen Akten $\mathrm{zu}$ dem Vorgang $\mathrm{zu}$ ermitteln sind. Eine Publikation aus dem Jahr 1858 bietet derzeit den frühesten Beleg für die Bernds-Schenkung und ist damit die zentrale Gewährsquelle für das Jubiläum 2018. Bis heute lassen sich im Bestand aber Brakteaten nachweisen, die mit großer Wahrscheinlichkeit aus dieser Schenkung stammen.

Aus dem 18. Jahrhundert sind vereinzelte weitere Schenkungen belegt. Die mit Abstand bedeutendste war das Vermächtnis des sächsischen Hofarztes Samuel Kretzschmar, das 1774 einging und neben einem numismatischen Buchbestand ca. 3.700 Münzen vor allem aus der Antike und dem 17./18. Jahrhundert umfasste. Diese Münzen wurden zusammen mit denen des Schatzfunds von Bernds in vier Schränken aufbewahrt. Da die Kretzschmar-Münzen drei Schränke einnahmen, lässt sich daraus auch eine Größenvorstellung der Bernds-Schenkung gewinnen, die demnach mehrere hundert Stück umfasst haben muss.

Bis ins mittlere 19. Jahrhundert verharrte die Münzsammlung auf diesem recht bescheidenen Niveau. Doch ab 1840 setzte unter dem seit 1833 amtierenden Bibliotheksdirektor Ernst Gotthelf Gersdorf eine äußerst dynamische Entwicklung ein, die innerhalb von 20 Jahren den Wandel zu einem großen Münzkabinett von überregionaler Bedeutung bewirkte. Als Historiker und numismatischer Spezialist engagierte sich Gersdorf intensiv für den Ausbau der Sammlung. Er setzte nicht nur ab 1841 einen eigenen Erwerbungsetat für Münzen durch, sondern erreichte 1851 auch den Ankauf der herausragenden Mittelaltersammlung des Leipziger Patriziers und namhaften
Numismatikers Carl Friedrich von Posern-Klett mit ihren über 45.000 bzw. nach anderen Quellen sogar mehr als 51.000 Objekten. Der Sammlung Posern-Klett verdankt sich bis heute die besondere Bedeutung der Leipziger Münzsammlung im Bereich der mittelalterlichen Münzen.

Weitere bestandserweiternde Erfolge kamen hinzu: Ab 1855 waren Münzfunde aus dem Leipziger Kreisdirektionsbezirk dem Kustos des Münzkabinetts vorzulegen, sodass Gersdorf, der schon seit dem Fund von Gerstenberg 1843 an solchen Stücken für die Sammlung interessiert war, bei seinen Ankäufen Erstzugriff auf das Material hatte. Die spektakuläre Erwerbung des 1856 in Paunsdorf bei Leipzig gefundenen Schatzes, der aus 1.018 ganzen und 639 halben Brakteaten von meist vorzüglichem Erhaltungszustand bestand, gehört in diesen Kontext. Ein umfangreicher Neuzugang vor allem im Bereich der antiken und neuzeitlichen Münzen folgte 1858 mit der testamentarisch vermachten Sammlung des königlich-sächsischen Finanzrats Ferdinand von Reiboldt, die ca. 26.500 Münzen umfasste.

Um 1860 war das Leipziger Münzkabinett damit auf ca. 75.000 bis 80.000 Objekte angewachsen und hatte bereits seine zeiten- und räumeübergreifende Ausrichtung erhalten. Zugleich wurde der Bestand in einem handschriftlichen Bandkatalog von schließlich 16 Bänden dokumentiert.

Unter Gersdorf wurde die Leipziger Münzsammlung auch ein wichtiger Akteur in der deutschen Numismatik. Gersdorf selbst war von 1865 bis zu seinem Tod 1874 Herausgeber der „Blätter für Münzfreunde“ und veröffentlichte hier 19 Aufsätze, die im Wesentlichen auf dem Bestand der Universitätsbibliothek basierten. Im Mai 1880 fand der erste Vereinstag der deutschen Münzforscher in Leipzig statt, einer der Initiatoren dieses Treffens war der neue Sammlungsdirektor Bruno Stübel. Auch nach Gersdorf konnte der Sammlungsbestand kontinuierlich ausgebaut werden. Die Erwerbung von kleineren Münzfunden und Partien größerer Funde wie denen aus Zwickau (1876), Seega (1902), Nordhausen (1911) oder Etzoldshain (1933) setzte sich fort. Bis zum Zweiten Weltkrieg wurde auch weiterhin gezielt auf Auktionen oder direkt von namhaften Numismatikern angekauft. Darüber hinaus gelang es immer wieder, größere Privatsammlungen zu übernehmen: 1872 die Sammlung Wilhelm Albert Müller mit 798 islamischen Münzen, 1925 ca. 2.000 Münzen des 19. und 20. Jahrhunderts aus dem Vermächtnis des Leipziger AnatomieProfessors Sandór Kästner, 1926 aus dem Vorbesitz des Sinologie-Professors August Conrady 176 ostasiatische Käsch-Münzen.

Der Zweite Weltkrieg brachte für die Sammlung, die seit 1922 von Bruno Hilliger geleitet wurde, eine einschneidende Zäsur. Bei der Auslagerung nach Schloss Mutzschen mussten die Münzen aus Platzgründen von den Tabletts genommen und zusammengepackt werden, womit die gesamte Sammlungserschließung aus hundert Jahren ver-

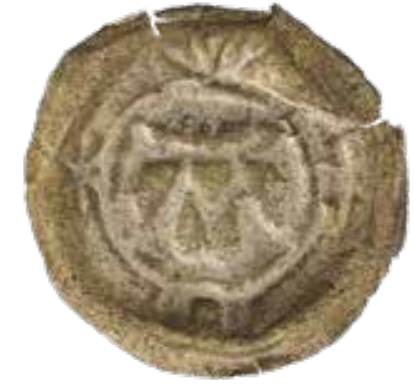

Pfennig (Brakteat), Bischof Albrecht II. von Mutzschen, 1258 bis 1266, Meißen ๑ UB Leipzig, Inv.nr. 1981/0895

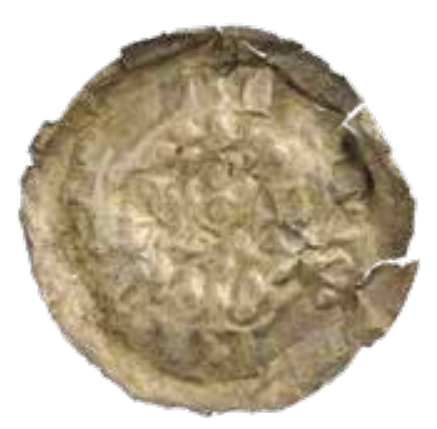

Pfennig (Brakteat), Bischof Bernhard von Kamenz oder Albrecht III. von Leisnig, 1293 bis 1312, Meißen Die beiden Stücke stammen mit hoher Wahrscheinlichkeit aus dem Münzfund von 1718, einem Brakteatenschatz mit Münzen aus dem 13. Jahrhundert. ๑ UB Leipzig, Inv.nr. 1981/0915 

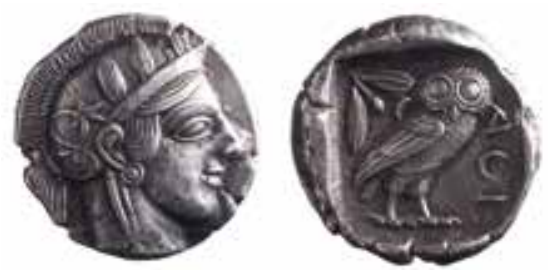

Tetradrachme, 450 bis 400 v. Chr., Athen Dieser Münztyp zeigt das hohe ästhetische Können der frühen griechischen Münzmeister. Die athenischen Münzen mit der Eule waren in der Antike allgemein und überregional anerkanntes Zahlungsmittel und wurden zum Teil auch von anderen Staaten imitiert. Die sprichwörtliche Redensart „Eulen nach Athen tragen", die auf den griechischen Dichter Aristophanes zurückgeht, dürfte sich auf diese Geldstücke beziehen und ist vor dem Hintergrund des damaligen Reichtums der Stadt zu verstehen.

๑ UB Leipzig, Inv.nr. 2017/8925

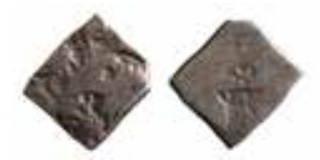

Karshapana, 320 bis 185 v. Chr., Maurya-Reich (Indien) Die zum Teil mehreckigen oder runden Silberstücke des Maurya-Reichs finden sich in ganz Indien in Schatzfunden. Das Silber wurde mit verschiedenen Punzen gestempelt, um die offizielle Legitimation zu erhalten. Die Deutung der einzelnen Symbole ist noch immer nicht abgeschlossen und stellt ein faszinierendes Forschungsgebiet dar.

๑ UB Leipzig, Inv.nr. 2014/1831
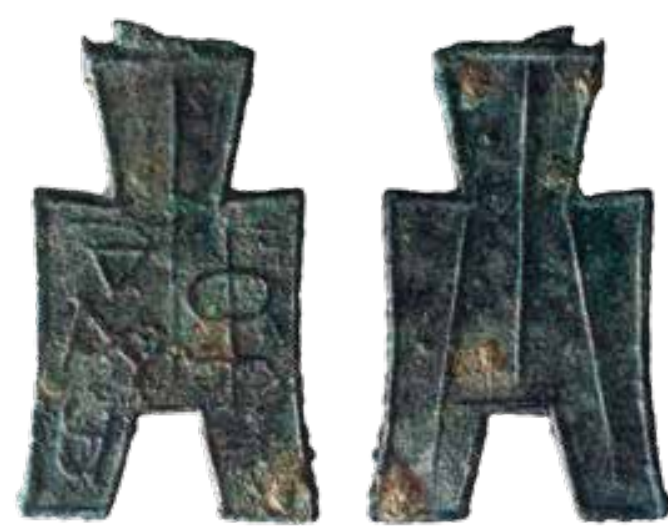

Spatengeld, 350 bis 250 v. Chr., Zhao-Staat (nördliches China)

(Abbildung verkleinert)

In China etablierten sich in der Frühzeit Münzen in Form von Gebrauchsgeräten. Die bekanntesten sind Spatenund Messergeld, die sich neben frühen Rundmünzen ab ca. 650 v. Chr. als Geldform entwickelten. Erst ab 221 v. Chr. wurde rundes Münzgeld im erstmals vereinten chinesischen Kaiserreich als einheitliches Zahlungsmittel eingeführt.

๑ UB Leipzig, Inv.nr. 2015/1933

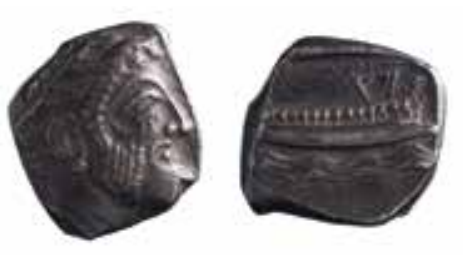

Stater, 400 bis 350 v. Chr., Arados (Phönizien) Die phönizischen Münzen sind $u$. a. eine wichtige Quelle für die phönizische Schrift. Hier befindet sie sich auf der Rückseite oberhalb der Galeere.

๑ UB Leipzig, Inv.nr. 2017/9507

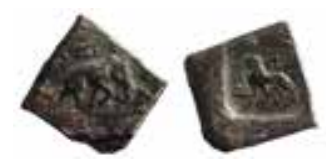

11/2 Karshapana, 185 bis 160 v. Chr., Taxila (Nordindien) Taxila war ein Schmelztiegel der Kulturen am Fuße des Himalajas. Man vermutet hier eine Keimzelle des damals noch unabhängigen indischen Münzwesens. Bereits Alexander der Große nahm die Stadt kampflos ein, was dazu führte, dass griechische und indische Kultur friedlich nebeneinander existieren konnten. Das Leipziger Stück zeigt mit Elefant, Löwe, dem dreihügeligen Berg und der Swastika typische indische Symbole.

๑ UB Leipzig, Inv.nr. 2015/0930

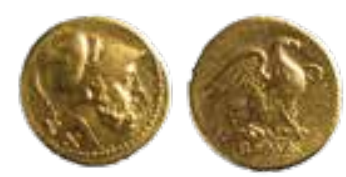

60 Asse, 211 bis 209 v. Chr., Rom

Goldmünzen wurden in der Zeit der Römischen Republik selten ausgegeben. Die frühen Emissionen goldener Vielfache des römischen As stehen meist in Zusammenhang mit großen Staatsausgaben. Dieses Stück aus der Zeit des Zweiten Punischen Krieges diente möglicherweise zur Begleichung der enormen Kriegskosten. Erst mit der weiteren wirtschaftlichen Entwicklung wurde die spätere römische Goldmünze, der Aureus, ein extrem wichtiges Nominal für den Großhandel.

○ UB Leipzig, Inv.nr. 1996/0431 


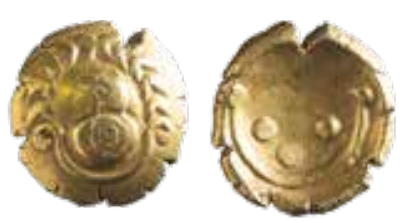

Stater, 2. bis 1. Jahrhundert v. Chr., Germanien Die Stater oder auch Regenbogenschüsselchen genannten Münzen wurden in verschiedenen Metallen (Gold, Silber, Elektron) im Bereich des Rheinlandes bis nach Ungarn von den unterschiedlichen germanischen Volksstämmen hergestellt und als Münzgeld eingesetzt. ๑ UB Leipzig, Inv.nr. 2017/8059
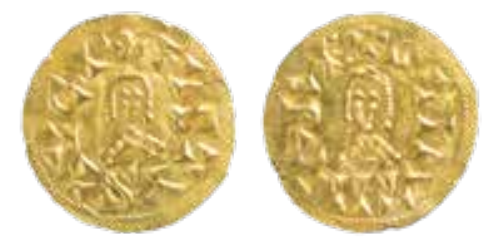

Tremissis, Suinthila, König der Westgoten, 621 bis 631, Tucci (Spanien)

Die Westgoten schufen sich in der Völkerwanderungszeit nach dem Zusammenbruch des Römischen Reiches auf der iberischen Halbinsel eine neue Heimat. Sie begannen, ein eigenes Münzsystem zu etablieren. Es basierte ausschließlich auf Gold. Die Münzen ahmten den byzantinischen Solidus nach.

๑ UB Leipzig, Inv.nr. 1996/0433
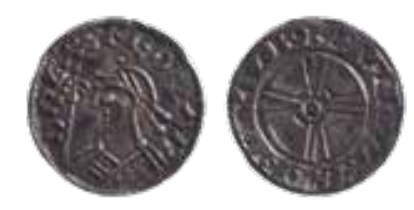

Penny, König Edward der Bekenner, 1050 bis 1053, England

Der Penny ist die englische Version des karolingischen Denars. Seit dem 10. Jahrhundert unterstand das Münzrecht ausschließlich dem König, was sich in der englischen Geschichte nicht änderte. Anders verlief es in den anderen Gebieten des europäischen Kontinents. Hier schafften es Abteien, Bistümer, Grafen und andere Adlige, sich das Münzrecht übertragen zu lassen und es in eigener Regie auszuüben.

๑ UB Leipzig, Inv.nr. 2009/0711

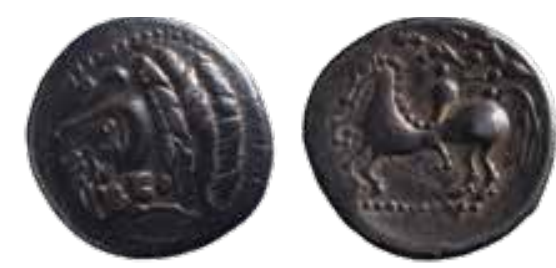

Stater, 1. Jahrhundert v. Chr., Ungarn

Die unter dem Begriff „Kelten“ zusammengefassten Völker imitierten das Geld der kulturell vorbildlichen griechischen Welt. Bei dem hier abgebildeten Stück handelt es sich um die Nachahmung einer Tetradrachme Philipps II. von Makedonien.

๑) UB Leipzig, Inv.nr. 2017/8076

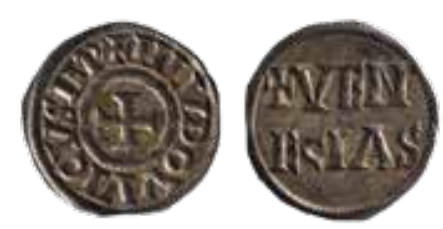

Denar, Kaiser Ludwig der Fromme, 819 bis 822, Venedig Unter Karl dem Großen wurde das Münzwesen im karolingischen Reich neu geordnet. Die Vereinheitlichung von Gewicht und Reinheitsgehalt des Silbers ermöglichten einen wirtschaftlichen Aufschwung. Die qualitativ hochwertigen Münzen wurden auch noch mehrere Jahrzehnte nach Karls Tod unter seinem Sohn und seinem Enkel weitergeprägt, bis die Normen wieder aufgeweicht wurden und die Qualität der Münzen sich schrittweise verringerte.

๑) UB Leipzig, Inv.nr. 2000/0072
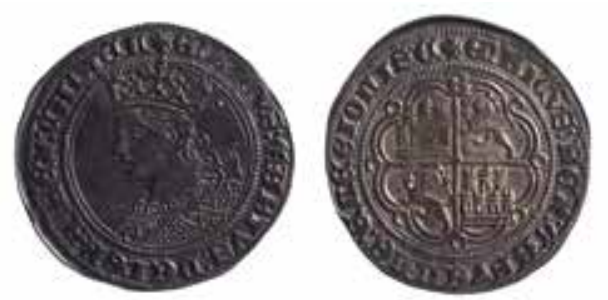

Real, König Heinrich IV. von Kastilien und Leon, 1454 bis 1474, Spanien

Der Real wurde erstmals als Goldmünze im

14. Jahrhundert ausgegeben und später auch als Silbermünze. Aufgrund der enormen Silberlieferungen aus den amerikanischen Kolonien, die in Spanien als Reals ausgemünzt wurden, lief der Real fast weltweit als akzeptiertes Münzgeld um. Insbesondere im 18. Jahrhundert waren die 8-Real-Münzen eine internationale Handelsmünze, die in Arabien oder China allgemein akzeptiert war.

๑ UB Leipzig, Inv.nr. 2013/0096 

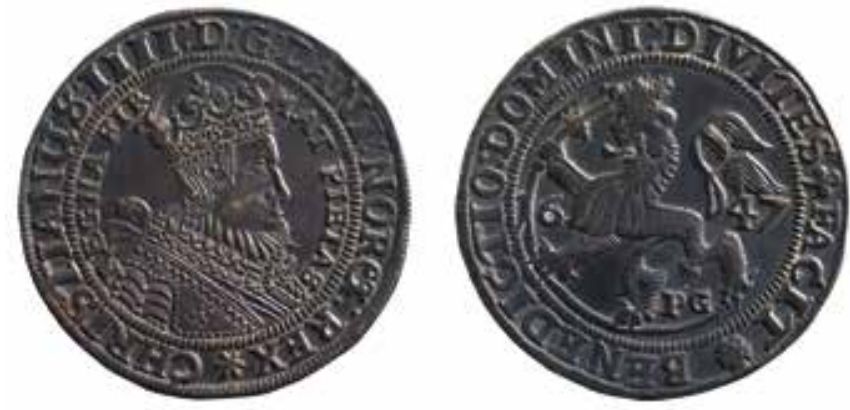

Speciesdaler, König Christian IV. von Dänemark und Norwegen, 1647, Norwegen Die erstmals Ende des 15. Jahrhunderts vereinzelt gefertigten Großsilbermünzen, die ursprünglich dem Gold-Gulden entsprachen, verbreiteten sich innerhalb kurzer Zeit in ganz Europa und Übersee. Die schweren silbernen Münzen mit verschiedensten Bezeichnungen (Taler, Daler, Dollar, Peso etc.) waren bis zum Beginn des 20. Jahrhunderts wesentlicher Bestandteil des allgemeinen Geldumlaufs.

๑ UB Leipzig, Inv.nr. 2014/2249
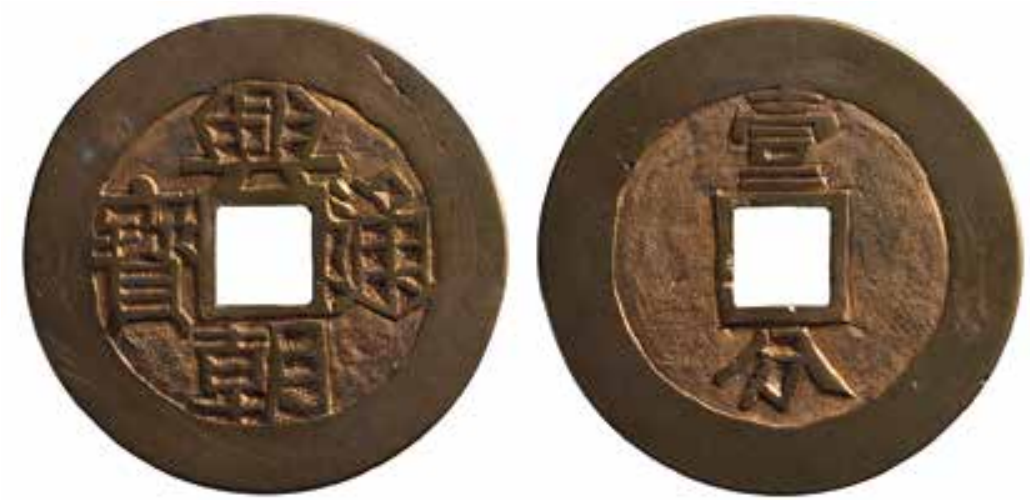

1 Fen, Sun-Kewang, 1647 bis 1657 , China

Seit 221 v. Chr. waren die runden Münzen mit quadratischem Loch, genannt Käsch/Cash, das einzige Zahlungsmittel in China. Bis zur Mitte des 19.

Jahrhunderts sind höhere Nominale der Cash-Münzen sehr selten. In der kriegerischen Umbruchszeit von der Ming-zur Qing-Dynastie setzten sich mehrere Prinzen der Ming-Dynastie in kleine Herrschaftsgebiete ab. Von dort bekämpften sie als Rebellen die neuen Herrscher. Wahrscheinlich zur Finanzierung der Kriegskosten gaben sie oft höhere Nominale aus, wie diesen Fen, der als 10 Cash galt.

๑ UB Leipzig, Inv.nr. 2011/0125
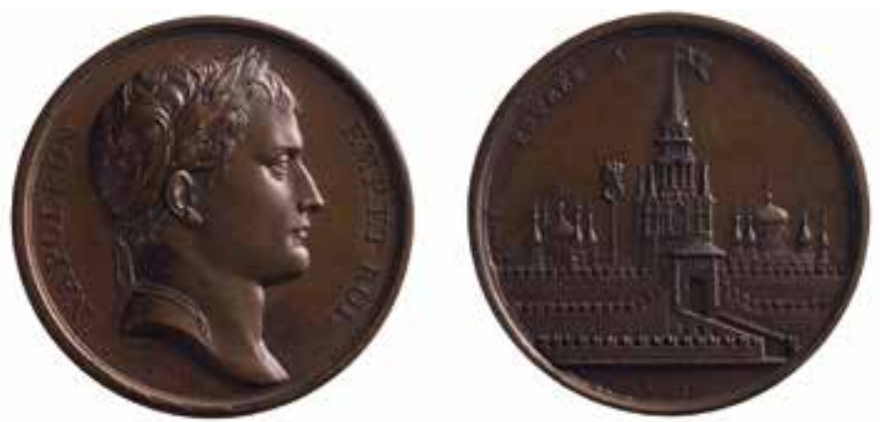

Medaille auf die Einnahme von Moskau, Kaiser Napoleon I., 1812, Frankreich Wie kein anderer setzte Napoleon Bonaparte (1769-1821) Medaillen als Propagandamittel ein. Er ließ auf alle seine Erfolge Medaillen fertigen. Ein Beispiel ist dieses Stück auf die Einnahme Moskaus. Besonders symbolträchtig sind die dargestellte Napoleon-Standarte auf der Stadtmauer und die Trikolore auf dem Stadttor vor dem Kreml. Sie machen den militärischen Erfolg unmittelbar anschaulich. Die Medaillenserie Napoleons war derartig beliebt, dass die Stücke mehrfach nachgefertigt wurden, um die Nachfrage zu befriedigen.

๑ UB Leipzig, Inv.nr. 2012/0858
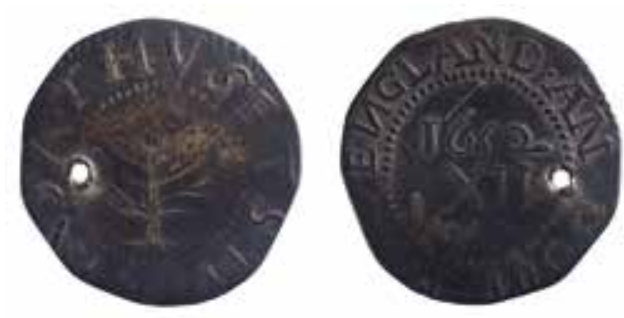

1 Shilling, 1652, Massachusetts (USA)

Mit dem Aufblühen der amerikanischen Kolonien wurden auch hier vermehrt Münzen benötigt, um den Handel weiter zu entwickeln. Zunächst noch mit Erlaubnis des Mutterlandes begannen auch die europäischen Siedler in Nordamerika, ihre eigene neue Identität auf Münzen abzubilden, und dokumentierten insbesondere zur Mitte des 18. Jahrhunderts damit ihren Willen zur Unabhängigkeit.

๑ UB Leipzig, Inv.nr. 2015/5002
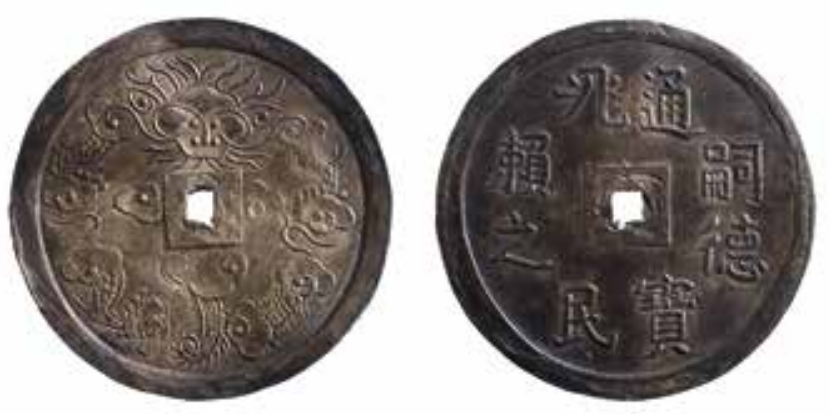

5 T'ien, Kaiser Tự Đức, 1848 bis 1883, Annam

Die aus China bekannten Lochmünzen strahlten in ihrem Umlaufgebiet auf den gesamten Bereich Südostasiens aus. Auch in Annam (heute Vietnam) wurden seit frühester Zeit Cash-Münzen ausgegeben. Größere Zahlungen wurden in Silberbarren getätigt. Mitte des 19. Jahrhunderts wurden in geringer Anzahl auch große Silbermünzen mit quadratischem Loch ausgegeben. Wahrscheinlich dienten diese Stücke jedoch eher der Auszeichnung von verdienten Beamten und nicht als Gebrauchsmünze für den Handel.

○ UB Leipzig, Inv.nr. 2011/0410
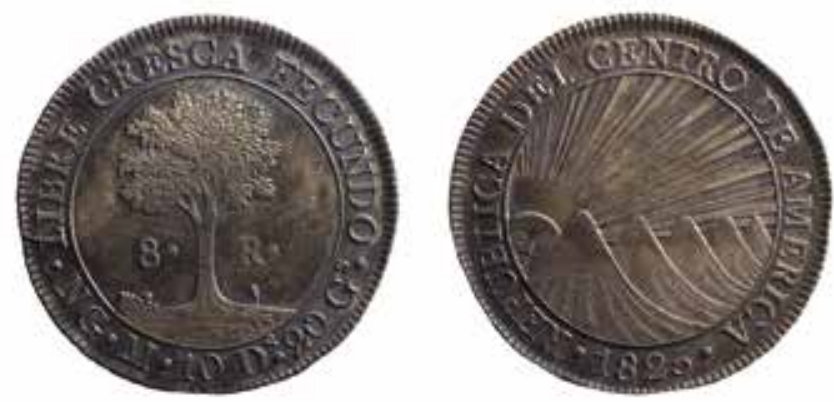

8 Reals, 1825, Zentralamerikanische Republik

Als sich im 19. Jahrhundert die zentral- und südamerikanischen Kolonien ihre Unabhängigkeit erkämpften, ging mit der Staatenbildung auch die Herausbildung einer eigenständigen Münzemission einher. Es entstanden vielfältige Münzbilder, die auch die Identitätsfindung der neuen Staaten durch eine entsprechende Ikonographie und nationale Symbole unterstützen sollten. Neben aller Vielfalt - auch in Hinblick auf die Namen der neuen Währungen (Sol, Peso, Boliviano etc.) - blieb jedoch der Silber- und Gewichtsstandard annähernd konstant und orientierte sich an den zuvor in Südamerika geschlagenen spanischen Münzen. ○ UB Leipzig, Inv.nr. 2017/2552 
nichtet wurde. Der Bestand an Goldmünzen, der separat in Schließfächern der Deutschen Bank in Leipzig untergebracht war, ging offenbar nach dem Einmarsch der US-Truppen verloren. Die in Mutzschen gelagerten Bestände wiederum wurden von der Roten Armee nach Leningrad und wohl auch nach Moskau abtransportiert, wo erste Neuordnungen und -bestimmungen begannen. 1958 erfolgte zwar die Rückgabe an die DDR, die Münzen verblieben aber zunächst am Münzkabinett in Ost-Berlin. Erst 1964 gelang es der Leipziger Universität, die Sammlung zurückzuerhalten. Bei der Ankunft in Leipzig war sie von weit über 90.000 auf ca. 81.000 Stücke geschrumpft.

Es dauerte vier weitere Jahre, bis 1968 unter Arnold Roggisch die Wiederaufarbeitung des Sammlungsbestandes begann. Da die Universität über keine Numismatiker verfügte, wurden hierfür Münzsammler als Ehrenamtliche angeworben. Die Wiedererschließung der Leipziger Sammlung war und ist daher mit zahlreichen Namen der sächsischen Numismatik verbunden: Citizen Science in seiner besten Ausprägung, lange bevor sich dieser Modebegriff etablierte. Neben Günther Röblitz und Günther Breitner begann damals auch Klaus Thieme mit der Sortierungsund Bestimmungsarbeit, der sein Engagement für die Sammlung bis 2010 fortsetzte und auf der Basis seiner Bestandskenntnis wichtige Veröffentlichungen zu den mitteldeutschen Brakteaten vorlegte. Seit den 1970er Jahren war Ewald Hausmann Teil des freiwilligen Münzteams, der ebenfalls bis 2010 intensiv für die Sammlungserschließung tätig war und sie mit Publikationen begleitete.

Unter Kustos Roland Jäger wurde ab 1978 die Wiedererschließung forciert. Mit dem Katalog der autonomen griechischen Münzen erschien 1993 ein erster publizierter Bestandsnachweis. Darüber hinaus erfolgten nun wieder Bestandsergänzungen: Durch Ankäufe konnte der kriegsbedingte Verlust der Goldmünzen abgemildert werden, die Kriegsverluste im Bereich der Groschen wurden durch die Erwerbung der Sammlung Günther Röblitz wenigstens teilweise kompensiert. 1999 erhielt das Kabinett die Münzen des Leipziger Silberschmiedes Treusch als Schenkung von dessen Frau, wodurch der Bestand der sächsischen Taler und der deutschen Münzen des 19. und 20. Jahrhunderts ergänzt werden konnte. Der entscheidendste Fortschritt aber war, dass die Sammlung 1998 im Zuge des Wiederaufbaus der teilweise kriegszerstörten Bibliothek eine neue, angemessene Unterbringung erhielt.

In den 2000er Jahren verlagerte sich mit dem nächsten Kustodenwechsel der Schwerpunkt der Sammlungsarbeit darauf, die Bestände öffentlich besser verfügbar zu machen. Mehrere neue Bestandskataloge konnten im Zuge der weiterhin ehrenamtlichen Erschließungstätigkeit publiziert werden: zu den provinzialrömischen Prägungen, den meißnischen Brakteaten, den sächsi-

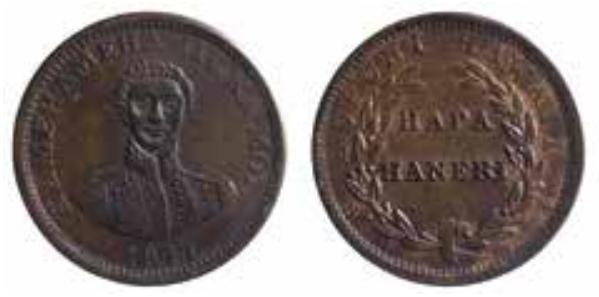

1/2 Hapa Haneli, König Kamehameha III., 1847, Hawaii In Hawaii zirkulierten zu Beginn des 19. Jahrhunderts US-Münzen und private Emissionen. Um die Münzgeldknappheit zu beenden, entschied sich der hawaiianische König, eigene Münzen in Umlauf zu geben.

๑ UB Leipzig, Inv.nr. 2018/2043
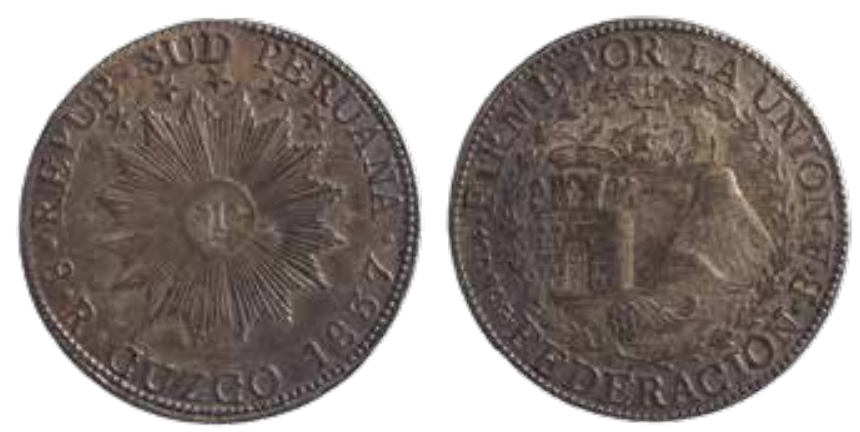

8 Reales, 1837, Republik Süd-Peru

Die Republik Süd-Peru bestand lediglich von 1836 bis 1839. Die Sonne nimmt Bezug auf den Herrscherkult der Inkas. Die Rückseit zeigt einen Teil der Inka-Ruinen Sacsayhuamán, ebenfalls als Verweis auf die Geschichte des Landes. Das goldene Füllhorn symbolisiert die Bodenschätze. Der Vulkan steht stellvertretend für die einzigartige Landschaft Perus. Das Segelschiff repräsentiert die 1821 gegründete Marine, die im Unabhängigkeitskrieg gegen Spanien eine wichtige Rolle spielte und sicherlich auch in dieser Zeit des Umbruchs die militärische Stärke darstellen sollte.

○ UB Leipzig, Inv.nr. 2018/0493

schen Groschen und den Leipziger Universitätsmedaillen. Seit 2015 kann sich die Münzsammlung der UB Leipzig dank dem Landesdigitalisierungsprogramm des Freistaates Sachsen am zentralen deutschen Münzportal KENOM (www.kenom.de) beteiligen und dort ihre Bestände sukzessive digital bereitstellen. Ein gutes Siebtel der Sammlung ist inzwischen bereits online.

\section{Ein Museum der internationalen Geldgeschichte}

Die Münzsammlung der Universitätsbibliothek Leipzig bietet numismatische Objekte aus nahezu allen Gegenden und Epochen der Geld- und Medaillengeschichte. Diese universelle Ausrichtung sollte und soll Forschung, Lehre und interessierter Öffentlichkeit für verschiedenste Fragestellungen dienen, insbesondere zu Münzentwicklung, Geldwesen, Handelsbeziehungen, Wirtschafts- und Sozialgeschichte oder historischen Repräsentationsformen. Münzen sind hierzu besonders gut geeignet, da es sich um authentische Quellen ihrer jeweiligen Zeit 


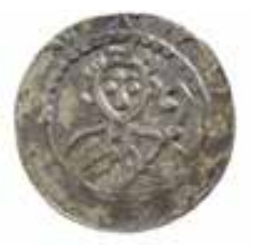

Pfennig (Brakteat), Abt Windolf oder Wiprecht von Groitzsch, um 1120, Pegau

Diese Münze stammt aus dem Fund von Gerstenberg, der 1843 gehoben wurde. Die erste Fundanalyse erfolgte durch den damaligen Kustos der Münzsammlung. Von diesem Typ sind derzeit nur zwei Stücke bekannt - beide liegen in der Sammlung der UB Leipzig. Die andere Variante zeigt ein ähnliches Münzbild, jedoch wurde dort der Krummstab durch eine Fahnenlanze getauscht. ๑ UB Leipzig, Inv.nr. 1979/0057

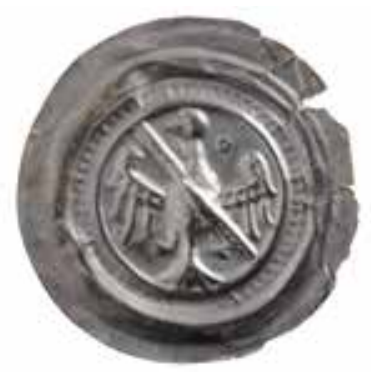

Pfennig (Brakteat), Vogt Heinrich I., 12./13.Jahrhundert, Straßberg

Im 13. Jahrhundert entstand eine Fülle von Prägestätten, die den aufblühenden Handel mit neuem Münzgeld unterstützten. Zu ihnen gehörte die Münzstätte Straßberg in der Nähe von Plauen. ๑ UB Leipzig, Inv.nr. 1987/0994 handelt und sie eine Vielfalt von Informationen bereitstellen, die sich auf anderen Wegen nicht oder oft nur ausschnitthaft erhalten haben.

An den Objekten der Leipziger Sammlung lassen sich zentrale Stationen der numismatischen Entwicklung nacherzählen.

Der Ursprung von Münzen als eine Form von Bezahlgeld geht weit zurück. Bereits die Sumerer im 3. Jahrtausend v. Chr. nutzten abgewogenes Silber nach einheitlichem Gewichtsmaß als Tauschmittel bei Handelsgeschäften. Normierte Metallobjekte, die in ihrer Formgebung oft hochwertige Tauschwaren nachahmten, sind aus der Übergangszeit vom Tausch- zum Geldhandel auch aus den chinesischen Gebieten der vorchristlichen Jahrhunderte und der frühen römischen Republik bekannt.

Aus der Verwendung von solchem „Gewichtssilber" entwickelten sich in Kleinasien im 7. Jahrhundert v. Chr. die ersten Münzen im eigentlichen Sinn. Auch bei ihnen war eine bestimmte und einheitliche Menge von Edelmetall zentrale Voraussetzung. Allerdings kam eine entscheidende Neuerung hinzu: die Prägung eines Münzbildes. Das Münzbild symbolisierte das Herstellungsmonopol des Herrschers und verdeutlichte seine Garantie für Menge und Reinheit des Edelmetalls. Die Vorteile dieses Tauschmittels waren enorm: Das staatlich verbürgte Gewicht und die Materialreinheit machte die Münze zu einem allgemein anerkannten Handelsgut, das nicht verderben konnte, überlokal eintauschbar war und sich insbesondere im Fernhandel für das unaufwendige Mitführen großer Werte bestens eignete.

Entsprechend große Bedeutung gewann diese Neuentwicklung schnell weit über Kleinasien hinaus. Mit der griechischen Kolonienbildung verbreitete sich das Münzsystem im gesamten antiken Mittelmeerraum. Die damit angestoßene Entwicklung setzte sich zunächst im europäischen und westasiatischen Bereich fort, mit der Ausbreitung des Islam und der kolonialen und imperialen Expansion der europäischen Staaten später auch weltweit, und wirkt bis zur heutigen Zeit nach. Vermutlich aus den Handelskontakten des Persischen Reiches mit den frühen Staaten auf dem Gebiet des heutigen Pakistan und Nordwestindiens gelangte die Idee des normierten Gewichtssilbers auf den indischen Subkontinent. Dort entwickelte sich im 5. Jahrhundert v. Chr. zunächst ein System von kleinen Silberbarren, meist im Gewicht von ca. 11 Gramm (= 1 Schekel oder 100 Ratti nach indischer Gewichtsnorm), die eine Prägung aufweisen. Aus ihnen entstand ein vollständig eigenes Münzsystem. Durch die Eroberungen Alexanders des Großen kam es zu Kontakten mit dem bereits weiterentwickelten griechischen Münzwesen, was sich auf die Gestaltung der Münzen in Indien nach und nach auswirkte. Später gelangte das indische Münzwesen unter den Einfluss der Sassaniden und der islamischen Herrscher, welche ihrerseits von Griechen, Römern und Byzantinern europäisch beeinflusst waren. Im Zuge der portugiesischen und britischen Kolonisierung verstärkte sich die Ausrichtung an europäischen Vorbildern. Spätestens ab dem 19. Jahrhundert unterschieden sich die indischen Münzbilder nur noch gering von denen westlicher Prägungen.

Ein weiterer Hotspot der Münzentwicklung war China. Dort bildete sich im 7. Jahrhundert ein vielseitiges Münzwesen aus. In den einzelnen Gebieten kamen dabei verschiedene Typen von Gerätegeld auf, das sich in seinen Formen an regional dominierenden Gerätschaften ausrichtete. So etablierte sich im Bereich Zentralchinas aus dem Spaten für den Feldbau Spatengeld in vielfältigen Varianten. Weiter nördlich, wo Viehzucht und Jagd die bestimmenden Wirtschaftsformen waren, wurde Messergeld verwendet. Im Süden war, neben den ohnehin in Ostasien allgemein akzeptierten Kauri-Muscheln, eine Goldwährung in Gebrauch. Im westlichen China entwickelte sich aus wertvollen Jadescheiben, die insbesondere als Ritualgeräte dienten, spätestens im 4. Jahrhundert v. Chr. eine kupferne Rundmünze mit einem Loch. Mit der Eroberung ganz Chinas und der Begründung des Kaiserreiches unter der Qin-Dynastie (221-207 v. Chr.) wurde die nun runde Münze mit einem quadratischen Loch die einheitliche Währung Chinas. Die später als Cash/Käsch bezeichnete Münze, die dem heutigen Bargeld seinen vermeintlich englischen Namen gab, verbreitete sich weit über die Grenzen Chinas hinaus in ganz Ostasien. Unter dem Einfluss der westlichen Mächte am Ende des 19. Jahrhunderts erfolgte auch hier eine Umstellung des bis dahin eigenständigen Münzsystems nach europäischem Vorbild.

Die Verwendung von Münzgeld hat sich in dieser mehr als 2.500-jährigen Geschichte über nahezu alle besiedelten Regionen der Erde ausgebreitet. Dabei hat sich eine beeindruckende Fülle an Typen und Gestaltungsformen herausgebildet. Indem jeder Kulturraum und jede Epoche jeweils eigene Ausprägungen hervorgebracht hat und sich unterschiedliche Zivilisationen wechselseitig beeinflusst haben, ist eine nahezu unüberschaubare Reihe individueller Zeugnisse entstanden, von denen jedes in eine einmalige historische Situation führt. Jede Münze ist daher auch eine einzigartige historische Quelle, nicht nur für das Selbstverständnis der jeweiligen Münzherren, sondern auch für wirtschaftliche und soziale Entwicklungen, politische Ereignisse, künstlerische Trends, handwerkliche Prozesse und gesellschaftlich prägende Vorstellungswelten. Das Münzkabinett der UB Leipzig bietet hiervon einen geradezu überreichen Fundus.

\section{Zum Medaillenbestand und einer bedeutenden Neuerwerbung}

Neben den Münzen entwickelte sich ab dem 15. Jahrhundert von Italien aus eine weitere Form der Prägung, die aber keine monetäre Funktion 
hatte, sondern ausschließlich repräsentativen Zwecken diente: Die Medaille verewigte bedeutende Ereignisse und Personen und verbreitete sich als Medium innerhalb verhältnismäßig kurzer Zeit weltweit.

Der numismatische Bestand der UB Leipzig besteht trotz der Bezeichnung als „Münzsammlung“ $\mathrm{zu}$ einem nicht unbeträchtlichen Teil auch aus solchen Medaillen, die keine Zahlungsmittel waren. Mit ca. 8.500 Stück machen sie ein Zehntel des gesamten Sammlungsbestands aus. Wie die Münzen decken die Medaillen räumlich und zeitlich eine große Spannbreite ab, doch gibt es in der Sammlung auch regionale Schwerpunkte, insbesondere den sächsisch-brandenburgischen Raum und natürlich Leipzig selbst. Mindestens 500 Medaillen des Bestands sind nach derzeitigem Erschließungsstand auf Leipziger Persönlichkeiten und Ereignisse ausgebracht, hinzu kommen zahlreiche weitere Stücke, die von Leipziger Medailleuren stammen, aber auf Anlässe oder Personen außerhalb Leipzigs angefertigt wurden.

Zeitlich reicht das Leipzig-Segment im Medaillenbestand vom Beginn der Medaillenprägung im frühen 16. Jahrhundert bis in die aktuelle Gegenwart. Allerdings sind bislang nicht alle Zeiträume gleich gut vertreten gewesen. Gerade die Phase ab den 1920er Jahren und bis um 1970 war auffallend unterrepräsentiert. Der Grund hierfür liegt im Rückgang der Erwerbungstätigkeit während der Weltwirtschaftskrise und verstärkt dann unter dem NS-Regime und natürlich in der existentiellen Unterbrechung, die 1943 mit der Auslagerung begann und erst 1968 mit der Neubearbeitung der rückgeführten Bestände endete.

Umso bedeutender ist die Erwerbung einer umfassenden Medaillensammlung zum Schaffen des großen Leipziger Medailleurs Bruno Eyermann, die im Jubiläumsjahr 2018 dank engagierter Drittmittelförderung gelang.

Bruno Eyermann (1888-1961) war die bestimmende Gestalt unter den Leipziger Kunstmedailleuren in der ersten Hälfte des 20. Jahrhunderts. Seine künstlerische Ausbildung erhielt er ab 1905 an der Leipziger Akademie für graphische Künste und Buchgewerbe, der heutigen Hochschule für Grafik und Buchkunst (HGB). Dort war er ab 1919 auch als Dozent tätig. Von 1923 an entfaltete er als freischaffender Künstler in Leipzig eine reiche Medaillenproduktion, die sich durch eine außergewöhnliche zeichnerische Sicherheit und Realitätstreue auszeichnete und mit ihrer Ausrichtung an der Klassischen Moderne bzw. der Neuen Sachlichkeit schnell vorbildlich für die Leipziger Kunstmedaille wurde und noch die Medaillenkunst der DDR maßgeblich beeinflusste. Charakteristisch sind auch seine Experimente mit verschiedenen Formaten und Materialien.

Das Medaillenwerk Eyermanns setzt um 1909 ein und deckt damit die Zeit des späten Kaiserreichs, der Weimarer Republik, der NS-Zeit und der ostdeutschen Nachkriegsära ab, für die letzten beid- en Lebensjahre auch die westdeutsche Wirtschaftswunderphase, nachdem Eyermann 1959 mit seiner Familie nach Hanau übergesiedelt war. In dieser gut 50-jährigen Schaffensphase lässt sich eine stilistische Entwicklung von äußerst detailliert gearbeiteten Feingravuren mit recht flachem Relief hin zu einer deutlich großzügigeren Behandlung mit stärker hervortretenden plastischen Elementen beobachten. Gleichbleibend ist aber die ausgeprägte Grundhaltung realistischer Wirklichkeitswiedergabe, die ein Markenzeichen Eyermanns darstellt. Angesichts der zeithistorischen Brüche, die Eyermann erlebte, ist es gleichzeitig interessant zu verfolgen, wie der Künstler in seinen Arbeiten stets eine Distanz zu den jeweiligen politischen Machthabern wahrte, was ihm zugleich ermöglichte, in jedem der Systeme Aufträge zu erhalten.

Die neuerworbene Medaillen-Sammlung dokumentiert das Euvre Eyermanns in einzigartiger Weise. Sie umfasst mit 218 Objekten über 80 Prozent des bekannten Medaillenwerks und vermittelt damit einen exzellenten Überblick über die verschiedenen Schaffensphasen, die künstlerische Entwicklung, die Auftraggeber und die Anlässe der einzelnen Arbeiten. Nirgends sonst existiert eine auch nur annähernd umfangreiche und vollständige Eyermann-Kollektion mit so ausgesucht qualitätvollen Exemplaren in hervorragendem Erhaltungszustand. Besonders erwähnenswert sind 18 Silber-Stücke, die als separat hergestellte Widmungsexemplare extremen Seltenheitswert haben.

Die von der UB Leipzig übernommene EyermannMedaillen-Sammlung wurde über Jahrzehnte hinweg von dem Leipziger Graphiker und Numismatiker Klaus Thieme († 2013) aufgebaut. Ihm ist die UB Leipzig in besonderer Weise verbunden, da er, wie bereits erwähnt, fast 40 Jahre lang die Wiedererschließung ihrer Münzsammlung maßgeblich mitgetragen hat. Es war Klaus Thiemes Wunsch, dass seine Eyermann-Sammlung geschlossen erhalten bleiben und möglichst in einer öffentlichen Institution der weiteren Nutzung zur Verfügung stehen solle. Dass die Medaillen nun gerade in den Bestand eingehen, den er so engagiert mit aufgearbeitet hat und in dem damit eine empfindliche Lücke geschlossen werden kann, ist besonders erfreulich.

Möglich gemacht wurde dies erst durch das Zusammenwirken mehrerer Förderer, wobei die Ostdeutsche Sparkassenstiftung zusammen mit der Sparkasse Leipzig eine besonders prominente Rolle spielte. Weitere Unterstützung erhielt die UB Leipzig von der Kulturstiftung der Länder, der Fritz Rudolf Künker GmbH \& Co. KG Osnabrück und dem Verband der Deutschen Münzenhändler e. V. (VDDM). Sie haben der Münzsammlung der UB Leipzig zu ihrem 300-jährigen Jubiläum nicht nur ein herausragendes Geburtstagsgeschenk gemacht, sondern auch künftiger Beschäftigung mit der Leipziger Kunstmedaille eine wichtige Ausgangsbasis verschafft.

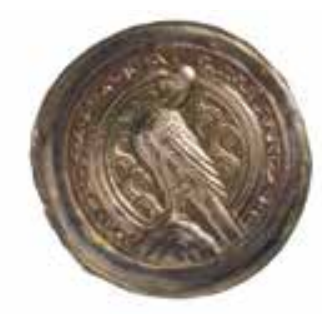

Pfennig (Brakteat), Burkhardt II., 1142 bis 1174, Ermsleben, Grafschaft Falkenstein Dieser Brakteatentyp ist ein Vertreter der extrem hohen Prägekunst, mit der gegen Ende des 12. Jahrhunderts Münzen hergestellt wurden. Mit den fein gearbeiteten, detaillierten Prägestempeln wurden eindrucksvolle romanische Kleinkunstwerke geschaffen. ๑ UB Leipzig, Inv.nr. 1987/1470

Literaturhinweise und weiterführende Informationen zur Leipziger Münzsammlung: Christoph Mackert (Hrsg.): Geldkulturerbe. 300 Jahre Münzsammlung der UB Leipzig, mit Beiträgen von Caroline Böhme, Sebastian Gensicke, Ivonne Kornemann u. a. (Schriften aus der Universitätsbibliothek Leipzig 41). Leipzig 2018.

\section{Autoren}

Dr. Christoph Mackert Universitätsbibliothek Leipzig Kustos der Münzsammlung mackert@ub.uni-leipzig.de

Thomas Uhlmann Ehrenamtlicher Mitarbeiter in der Münzsammlung der Universitätsbibliothek Leipzig 